ALEA, Lat. Am. J. Probab. Math. Stat. 16, 1089-1104 (2019)

DOI: 10.30757/ALEA.v16-40

\title{
Metastable behavior of bootstrap percolation on Galton-Watson trees
}

\author{
Assaf Shapira \\ Department of Mathematics and Physics, Roma Tre University \\ 1 Largo San Murialdo, \\ 00146 Roma Italy. \\ E-mail address: assaf shap@gmail.com \\ $U R L$ : https://assafshap.github.io
}

\begin{abstract}
We analyze the metastable behavior near criticality for bootstrap percolation on Galton-Watson trees. We find that, depending on the exact choice of the offspring distribution, it is possible to have several distinct metastable states, with varying scaling of their duration while approaching criticality.
\end{abstract}

\section{Introduction}

Bootstrap percolation is a deterministic dynamics in discrete time first introduced in Chalupa et al. (1979) in order to model disordered magnetic systems, and broadly studied since in many different contexts. Fix a graph $G$ and a parameter $r \in \mathbb{N}$. Each vertex of the graph can be in one of two states - infected or healthy, which are initially distributed independently with probabilities $p$ and $q=1-p$. At each time step we update these states, so that the infected vertices remain infected, and a healthy vertex becomes infected if it has at least $r$ infected neighbors. One may also consider more general infection conditions, such as the oriented bootstrap percolation - when the graph $G$ is oriented, and we require at least $r$ edges to point at infected vertices.

Bootstrap percolation on various deterministic graphs has been the subject of extensive research. On the grid $[n]^{d}$, the probability that all vertices are eventually infected, as a function of $p$ (or equivalently $q$ ), has been profoundly studied in Aizenman and Lebowitz (1988); Holroyd (2003); Balogh et al. (2012). For $(d+1)$ regular infinite trees, with $2 \leq r \leq d$, it is shown in Balogh et al. (2006) that a phase transition occurs. Defining $q_{c}$ to be the supremum over all $q$ such that starting with probability $q$ to be healthy all vertices end up being infected with probability 1 , an explicit expression for $q_{c}$ is found, and it is furthermore proven that $q_{c}$ lies in the

Received by the editors December 4th, 2018; accepted October 7th, 2019.

2010 Mathematics Subject Classification. 60K35, 60J80.

Key words and phrases. Bootstrap percolation, Metastability, Galton-Watson trees.

I acknowledge the support of the ERC Starting Grant 680275 MALIG. 
open interval $(0,1)$. In addition, it is determined, depending on $d$ and on $r$, when the transition is continuous and when it is discontinuous. In Biskup and Schonmann (2009) the details of the metastability properties are studied, describing the time evolution of the probability that the root stays healthy near criticality.

Random environments have also been of interest in this field, e.g., the bootstrap percolation on a polluted grid (Gravner and McDonald, 1997; Gravner and Holroyd, 2019), the random graph $G_{n, p}$ (Janson et al., 2012), the random regular graph (Balogh and Pittel, 2007; Janson, 2009), and the Galton-Watson tree (Bollobás et al., 2014).

In this paper, we will analyze the metastability of the bootstrap percolation on a directed Galton-Watson tree. The questions we address are analogous to those studied for regular trees in Biskup and Schonmann (2009), where the authors analyze the behavior of the infection probability close to the phase transition, that is, when $q=q_{c}-\epsilon$ for small (positive) $\epsilon$. They study the time dependence of the probability that the root stays healthy, that we denote by $\phi_{t}(q)$. By definition of $q_{c}$ whenever $q<q_{c}$ the limit of this probability at long times $\phi_{\infty}(q)=0$, but in some cases at $q_{c}$ this probability is strictly positive, and we say that the phase transition is discontinuous. In these cases, as $\epsilon$ tends to 0 , the behavior of $\phi_{t}(q)$ seems at first like its behavior at criticality, decreasing to the constant $\phi_{\infty}\left(q_{c}\right)$ and staying at this value for a long time. However, after some time it leaves the plateau and decays to 0 . They find that the length of the plateau scales as $\epsilon^{-1 / 2}$, and indeed at $q_{c}$ it becames infinite, explaining the discontinuity of the phase transition.

The behavior for the Galton Watson tree is much richer. In particular, we will prove that varying the offspring distribution can result in a multiple plateau behavior for $\phi_{t}^{\xi}$ the expected value (over the offspring distribution $\xi$ ) of the probability that the root is healthy. The main result, Theorem 3.8, gives an explicit construction of an offspring distribution that attains any possible choice for the widths of the multiple plateaux.

The structure of the paper is the following. In Section 2 we introduce the model. In section $\S 3.1$ we prove that $\phi_{t}^{\xi}$ corresponds to the limiting ratio of the number of infected vertices in a large ball around the root (Proposition 3.1). In section $§ 3.2$ we study the zoology of the metastabilities for different offspring distributions, and state our main results Theorem 3.5 and Theorem 3.8, showing that bootstrap percolation on Galton Watson trees has a vast variety of possible metastable behaviors. Finally, in section $\S 5$ we comment on other phase transitions that may occur and address some open questions.

\section{Model and notations}

Fix an infection threshold $r \geq 2$, and consider a Galton-Watson tree $G$ whose offspring distribution is supported on $r, r+1, \ldots$ That is, defining $\xi_{k}$ to be the probability that a vertex has $k$ children, we require $\xi_{k}=0$ for $k<r$.

In the beginning, we decide for each vertex of $G$ whether it is infected or healthy, independently with probabilities $p$ and $q=1-p$ respectively. Then, at each time step $t$, a healthy vertex will get infected if it has at least $r$ infected children. Let us denote by $\phi_{t}^{G}$ the probability that the root is healthy at time $t$, so in particular $\phi_{0}^{G}=q$. For $t>0$ this probability is a random variable, since it depends on the 
graph $G$. Note also that $\phi_{t}^{G}$ is decreasing in $t$. The expected value of $\phi_{t}^{G}$ over all graphs $G$, generated with offspring distribution $\xi$, will be denoted $\phi_{t}^{\xi}$.

One particular case, that has been studied in Balogh et al. (2006); Biskup and Schonmann (2009); Fontes and Schonmann (2008); Chalupa et al. (1979), is the case of a rooted $d$-ary tree, i.e., $\xi_{k}=\mathbb{1}_{k=d}$. Here, one can find $\phi_{t}^{d}$ recursively using the relation

$$
\begin{aligned}
\phi_{t+1}^{d} & =h_{d}\left(\phi_{t}^{d}\right) \\
h_{d}(x) & =q \mathbb{P}[\operatorname{Bin}(d, 1-x) \leq r-1] .
\end{aligned}
$$

For the GW tree, such a recursion still holds for the expected value $\phi_{t}^{\xi}$ :

$$
\begin{aligned}
\phi_{t+1}^{\xi} & =h_{\xi}\left(\phi_{t}^{\xi}\right) ; \\
h_{\xi}(x) & =\sum_{k=r}^{\infty} \xi_{k} h_{k}(x) .
\end{aligned}
$$

Remark 2.1. For convenience we have defined the model on the directed tree, where infections could only propagate in the direction of the root. As for the undirected tree, it has been observed in Biskup and Schonmann (2009) that the infection time of the root remains the same (deterministically for all initial conditions). Therefore, the main results of this article, theorems 3.5 and 3.8, will also hold for the infection time of the root in that case. Note, however, that Proposition 3.1 is concerned with the infection time of more sites other than the root, and adapting it to the undirected case is not immediate.

\section{Results}

3.1. Prevalence and $\phi_{t}$. Before stating the main results concerning the critical behavior of the bootstrap percolation we will state a small result that gives more motivation for the study of $\phi_{t}$. It is separate from the results of the next section, and will not be used in their proofs.

The relation in equation (2.3) allows us to find the expected value of $\phi_{t}^{G}$, but for a specific realization of $G, \phi_{t}^{G}$ may differ from that value. For example, fixing $t$, there is a nonzero probability that a finite neighborhood of the root will have many vertices of high degree, which will result in a smaller $\phi_{t}^{G}$. However, we will see that $\phi_{t}^{\xi}$ describes almost surely another observable - the prevalence, i.e., the limiting fraction of infected vertices. Namely, we define the $R$-prevalence at time $t$ as

$$
\rho_{R}(t)=\frac{\mid\{\text { infected vertices in } B(R) \text { at time } t\} \mid}{|B(R)|},
$$

where $B(R)$ denotes the ball of radius $R$ around the root.

It is natural to expect $\rho_{R}(t)$ to be close to $1-\phi_{t}^{\xi}$, and this is indeed the case, as shown in the following proposition:

Proposition 3.1. Fix $t$. Then $\lim _{R \rightarrow \infty} \rho_{R}(t)=1-\phi_{t}^{\xi}$ almost surely (in both the graph and the initial state measures). 
3.2. Critical behavior. Following Balogh et al. (2006); Bollobás et al. (2014), we define the critical probability

$$
q_{c}=\sup _{[0,1]}\left\{q: \phi_{\infty}^{\xi}=0\right\} .
$$

In order to analyze this criticality, define

$$
\begin{aligned}
& g_{k}(x)=\frac{h_{k}(x)}{q x}, \\
& g_{\xi}(x)=\frac{h_{\xi}(x)}{q x},
\end{aligned}
$$

for $h_{k}$ and $h_{\xi}$ defined in equations (2.2) and (2.4) respectively.

In Bollobás et al. (2014), the following fact is shown:

Fact 3.2. Fix $\xi$. Then:

(1) For a given $q, \phi_{\infty}^{\xi}$ is the maximal solution in $[0,1]$ of $g_{\xi}(x)=\frac{1}{q}$, and 0 if no such solution exists.

(2) $q_{c}=\frac{1}{\max _{[0,1]} g_{\xi}(x)}$.

The metastability behavior of the infection probability $\left(1-\phi_{t}^{\xi}\right)$ near the phase transition, when $q$ is slightly smaller than $q_{c}$, will be characterized using the following two definitions. They will describe the behavior illustrated in figure 3.1.

Definition 3.3. For $0<x<1$ and some positive $\delta$, the $\delta$-entrance time of $x$ is

$$
\tau_{x, \delta}^{-}(q)=\min \left\{t: \phi_{t}^{\xi}<x+\delta\right\}
$$

and the $\delta$-exit time is defined as

$$
\tau_{x, \delta}^{+}(q)=\min \left\{t: \phi_{t}^{\xi}<x-\delta\right\} .
$$

Definition 3.4. Fix $\delta>0$. We say that the critical point is $\delta$ - $\left(\nu_{1}, \ldots, \nu_{n}\right)$ metastable at $x_{1}>\cdots>x_{n}>0$ if, for $q \nearrow q_{c}$, the following hold:

(1) $\tau_{x_{1}, \delta}^{-}=O(1)$.

(2) $\frac{\log \left(\tau_{x_{i}, \delta}^{+}-\tau_{x_{i}, \delta}^{-}\right)}{\log \left(q_{c}-q\right)} \stackrel{q \nearrow q_{c}}{\longrightarrow}-1+\frac{1}{2 \nu_{i}}$ for $i=1, \ldots, n$.

(3) $\tau_{x_{i+1}, \delta}^{-}-\tau_{x_{i}, \delta}^{+}=O(1)$ for $i=1, \ldots, n$ and $x_{n+1}=0$.

We say that the critical point is $\left(\nu_{1}, \ldots, \nu_{n}\right)$-metastable at $x_{1}>\cdots>x_{n}$ if it is $\delta$ - $\left(\nu_{1}, \ldots, \nu_{n}\right)$-metastable at $x_{1}>\cdots>x_{n}$ for small enough $\delta$.

The following theorem gives a full classification of the metastability properties of the phase transition, using $g_{\xi}$ defined in equation (3.3).

Theorem 3.5. Fix $\xi$. Then the metastable behavior of $\phi_{t}^{\xi}$ at the phase transition is determined by one of the following cases:

(1) $g_{\xi}$ attains its maximum at 1 . In this case the critical probability is 1 .

(2) $g_{\xi}$ has a unique maximum at 0 . In this case the phase transition is continuous, i.e., $\phi_{\infty}^{\xi}=0$ at $q=q_{c}$. Then at the critical point

$$
g_{\xi}(x)=\frac{1}{q_{c}}-C x^{\nu}+o\left(x^{\nu}\right)
$$

for some $\nu \in \mathbb{N}$ and a positive constant $C$, and

$$
\frac{\log \left(\phi_{t}^{\xi}\right)}{\log t} \stackrel{t \rightarrow \infty}{\longrightarrow}-\frac{1}{\nu}
$$




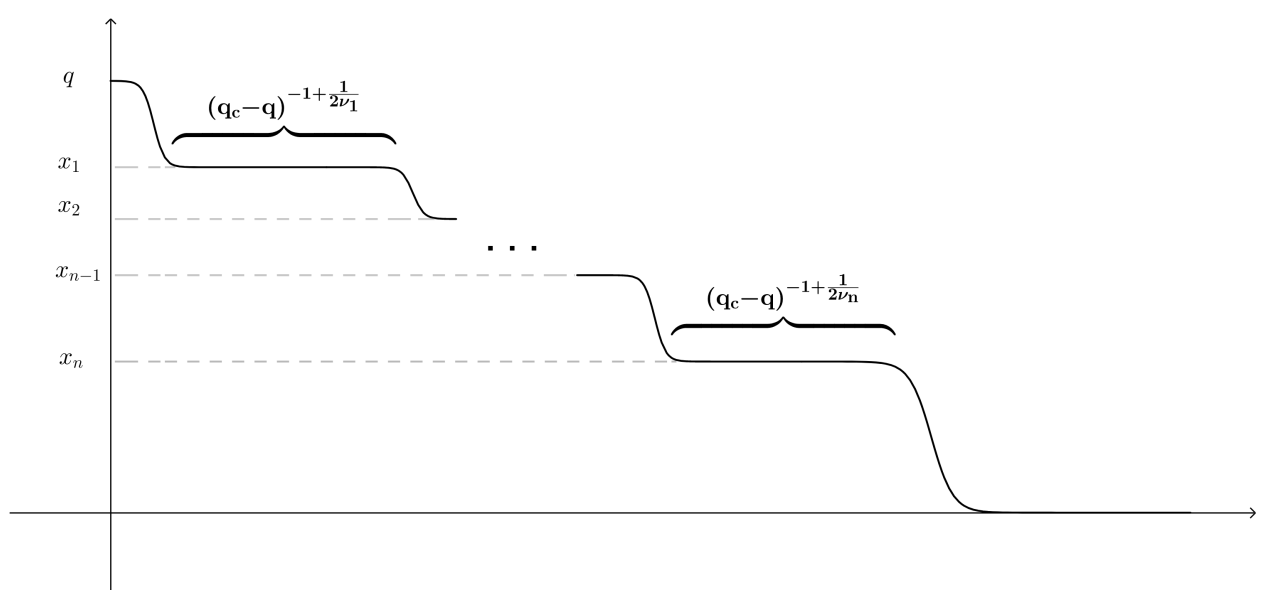

FIGURE 3.1. A schematic picture of $\phi_{t}^{\xi}$ as a function of $t$ for a $\left(\nu_{1}, \ldots, \nu_{n}\right)$-metastable criticality at $x_{1}>\cdots>x_{n}$.

(3) The maximum of $g_{\xi}$ is attained at the points $x_{1}, \ldots, x_{n}$ for $1>x_{1}>$ $\cdots>x_{n}>0$, and possibly also at 0 . In this case the phase transition is discontinuous. For $i=1, \ldots, n$ we may write around $x_{i}$

$$
g_{\xi}(x)=\frac{1}{q_{c}}-C_{i}\left(x-x_{i}\right)^{2 \nu_{i}}+o\left(\left(x-x_{i}\right)^{2 \nu_{i}}\right)
$$

for some $C_{i}>0$.

Then the critical point is $\left(\nu_{1}, \ldots, \nu_{n}\right)$-metastable at $x_{1}>\cdots>x_{n}$.

Remark 3.6. In the first case, where the critical probability is 1 , it is not clear whether or not an asymptotic expansion exists, since $g_{\xi}$ is not guaranteed to be analytic. When it does exist, one can recover a result similar to Case 3.

Remark 3.7. The position $x_{1}$ has a clear interpretation - it is the probability to stay healthy when $q=q_{c}$. As $q$ approaches criticality, the first plateau becomes longer and longer, and at $q_{c}$ it is infinite, explaining the discontinuity of the phase transition. The other positions $x_{2}, \ldots, x_{n}$, however, do not have such an interpretation.

We can now state our main result, proving that all the different metastable behaviors described above can be attained. Actually, the proof of Theorem 3.8 is constructive: we provide for any choice of the widths of the multiple plateaux, an offspring distribution which realizes the corresponding metastable behavior.

\section{Theorem 3.8.}

(1) Let $\nu \in \mathbb{N}$. Then there exists $\xi$ such that the phase transition is continuous, and satisfies equation (3.5) at criticality.

(2) Let $\left(\nu_{1}, \ldots, \nu_{n}\right) \in \mathbb{N}^{n}$. Then there exist $\xi$ and $x_{1}>\cdots>x_{n}$ such that the critical point is $\left(\nu_{1}, \ldots, \nu_{n}\right)$-metastable at $x_{1}>\cdots>x_{n}$.

Remark 3.9. The proof of the second part is perturbative, showing that an appropriate $\xi$ exists when $x_{1}, \ldots, x_{n}$ are small enough, and could be found by solving an explicit system of linear equations. A more general result that charecterizes the possible positions $x_{1}, \ldots, x_{n}$ in beyond the scope of this article. 


\section{Proofs}

Proof of Proposition 3.1: The idea of the proof is to notice that the main contribution to the prevalence comes from the sites close to the boundary, and then use their independence. Thus, we fix a width $w$, and consider

$$
\rho_{R, w}(t)=\frac{\mid \text { infected vertices in } B(R) \backslash B(R-w) \text { at time } t\} \mid}{|B(R) \backslash B(R-w)|} .
$$

First, we claim that $\rho_{R}(t)$ is approximated by $\rho_{R, w}(t)$ for large $w$. More accurately, we have $|B(R-w)| \leq 2^{-w}|B(R)|$, which also implies that the number of infected vertices in $B(R) \backslash B(R-w)$ is the same as the number of infected vertices in $B(R)$, up to a correction of order $2^{-w}|B(R)|$. Then

$$
\rho_{R}(t)=\rho_{R, w}(t)+O\left(2^{-w}\right) .
$$

We would now like to bound the distance between $\rho_{R, w}(t)$ and $1-\phi_{t}^{\xi}$. Let $\varepsilon>0$, and, by equation (4.1), take $w$ big enough such that $\left|\rho_{R}(t)-\rho_{R, w}(t)\right|<\frac{\varepsilon}{2}$ uniformly in $R$. Note that $\rho_{R, w}(t)$ is a weighted average of the $w$ random variables $\rho_{R, 1}(t), \rho_{R-1,1}(t), \ldots, \rho_{R-w+1,1}(t)$, and consider one of these variables, $\rho_{r, 1}(t)$. This variable is the average of the random variables $\mathbb{1}_{v \text { is infected }}$ for all vertices $v$ of distance $r$ from the root, and since these are independent Bernoulli random variables with mean $1-\phi_{t}^{\xi}$, and since there are at least $2^{R-w+1}$ such variables, we can use a large deviation estimate, yielding

$$
\mathbb{P}\left[\left|\rho_{r, 1}(t)-\left(1-\phi_{t}^{\xi}\right)\right|>\frac{\varepsilon}{2}\right] \leq e^{-c 2^{R-w+1}}
$$

for a positive $c$ that only depends on $\varepsilon$ and on $\phi_{t}^{\xi}$. Since for $1-\phi_{t}^{\xi}$ to be far from $\rho_{R, w}(t)$ it must be far from at least one of the variables $\rho_{R, 1}(t), \rho_{R-1,1}(t), \ldots$, $\rho_{R-w+1,1}(t)$, we have

$$
\mathbb{P}\left[\left|\rho_{R, w}(t)-\left(1-\phi_{t}^{\xi}\right)\right|>\frac{\varepsilon}{2}\right] \leq w e^{-c 2^{R-w+1}} .
$$

Hence, $\rho_{R}(t)$ is $\varepsilon$-close to $1-\phi_{t}^{\xi}$ with probability larger than $1-w e^{-c 2^{R-w+1}}$, which concludes the proof by the Borel-Cantelli lemma.

Before proving Theorems 3.5 and 3.8, we will need a couple of properties of the funtions $g_{k}$ defined in equation (3.2).

Claim 4.1. $g_{k}$ is a polynomial of degree $k-1$, whose lowest degree monomial is of degree $k-r$.

Proof: By equations (3.2) and (2.2)

$$
\begin{aligned}
g_{k}(x) & =\frac{\mathbb{P}[\operatorname{Bin}(k, 1-x) \leq r-1]}{x} \\
& =\sum_{i=0}^{r-1}\left(\begin{array}{c}
k \\
i
\end{array}\right)(1-x)^{i} x^{k-i-1} ;
\end{aligned}
$$

therefore all monomials are of degree between $k-r$ and $k-1$. The coefficient of $x^{k-r}$ is $\left(\begin{array}{c}k \\ r-1\end{array}\right) \neq 0$, and the coefficient of $x^{k-1}$ is $\sum_{i=0}^{r-1}\left(\begin{array}{c}k \\ i\end{array}\right)(-1)^{i}$, which is also nonzero since $0<r-1<k$. This concludes the proof.

Claim 4.2. $g_{r}(x), \ldots, g_{m}(x), x^{m-r+1}, \ldots, x^{m-1}$ is a basis of the linear space of polynomials of degree smaller or equal to $m-1$. 
Proof: Denote $v_{1}(x)=g_{r}(x), \ldots, v_{m-r+1}(x)=g_{m}(x), v_{m-r+2}(x)=x^{m-r+1}$, $v_{m}(x)=x^{m-1}$. By Claim 4.1, all $v^{\prime}$ 's are of degree smaller or equal to $m-1$. Moreover, the matrix whose $(i, j)$ entry is the coefficient of $x^{j}$ in the polynomial $v_{i}$ is upper triangular, with nonzero diagonal. This shows that $\left\{v_{i}\right\}_{i=1}^{m}$ is indeed a basis.

We will also use the following result from Bollobás et al. (2014):

Claim 4.3 (Claim 3.9 of Bollobás et al., 2014). Let $\xi=\left(\xi_{k}\right)_{k \geq 1}$ where $\xi_{k}=\frac{r-1}{k(k-1)}$. Then $g_{\xi}(x)=1$ for all $x \in[0,1]$.

We are now ready to prove Theorems 3.5 and 3.8 .

Proof of Theorem 3.5: First, we note that $g_{k}(1)=1$ for all $k$, so in particular the series $\sum_{k=r}^{\infty} \xi_{k} g_{k}(x)$ converges at $x=1$. By Claim 4.1, the monomials of degree up to $n$ of the partial sum $\sum_{k=r}^{N} \xi_{k} g_{k}(x)$ are fixed once $N>n+r$. This allows us to write $g_{k}$ as a power series, taking the coefficient of $x^{n}$ to be the one that appears in these partial sums. Hence, $g_{\xi}(x)$ is analytic in $(-1,1)$ and continuous at 1 , and thus cases 1,2 and 3 exhaust all possibilities.

The result will then follow from general arguments of dynamical systems near a bifurcation point. Since the exact calculations are a bit tedious, we only give here a short sketch of the argument, referring to the appendix for the complete proof (Theorem A.4 for case 2 and Lemma A.13 for case 3).

For case 2, recall equations (2.3), (3.3) and (3.4). These yield the expression

$$
\phi_{t+1}=\phi_{t}-C q_{c}\left(\phi_{t}\right)^{\nu+1}+o\left(\left(\phi_{t}\right)^{\nu+1}\right),
$$

that could be estimated by comparing to the differential equation

$$
\frac{\mathrm{d} \phi}{\mathrm{d} t}=-C q_{c}\left(\phi_{t}\right)^{\nu+1}
$$

This equation could be solved explicitly, obtaining the asymptotics of equation (3.5).

For case 3 equations (2.3), (3.3) and (3.6) yield, around $x_{i}$, the relation

$$
\phi_{t+1}=\phi_{t}-\frac{x_{i}}{q_{c}}\left(q_{c}-q\right)-C_{i} q x_{i}\left(\phi_{t}-x_{i}\right)^{2 \nu_{i}}+\text { error }
$$

with an error term which is negligible as $q \rightarrow q_{c}$ and $\phi_{t} \rightarrow x_{i}$. The approximate differential equation will be

$$
\frac{\mathrm{d} \phi}{\mathrm{d} t}=-\frac{x_{i}}{q_{c}}\left(q_{c}-q\right)-C_{i} q_{c} x_{i}\left(\phi-x_{i}\right)^{2 \nu_{i}}
$$

The solution of this equation has a plateau around $x_{i}$, whose length diverges as $\left(q_{c}-q\right)^{-1+\frac{1}{2 \nu_{i}}}$.

Finally, we should verify that the time spent between the plateaus (i.e., when $\left|\phi_{t}^{\xi}-x_{i}\right|>\delta$ for all $i$ ) in bounded uniformly in $q$. This could be proven by considering the function $A:\left[0, q_{c}\right] \times(0,1) \rightarrow[0,1]$ defined as

$$
A(q, x)=h_{\xi}(x)-x,
$$

where the dependence of the right hand side in $q$ is implicit (see equations (2.4) and (2.2)). This is a continuous function, and it only vanishes when $q=q_{c}$ and $x \in\left\{x_{1}, \ldots, x_{n}\right\}$ (see Fact 3.2, equation (3.3) and recall that $x_{1}, \ldots, x_{n}$ are the maxima of $g_{\xi}$ ). Therefore, choosing $\delta$ small enough, $A$ is strictly positive on the closed set $\left[0, q_{c}\right] \times\left(\left[\delta, x_{n}-\delta\right] \cup\left[x_{n}+\delta, x_{n-1}-\delta\right] \cup \cdots \cup\left[x_{1}+\delta, 1\right]\right)$. We can then choose 
$a$ to be its minimun over this set. This would give by equation (2.3) a decrease rate between the plateaus which is uniform in $q$, showing that the time spent there is bounded as $q \rightarrow q_{c}$.

Proof of Theorem 3.8 part 1: For the first part, according the Theorem 3.5, it will be enough to show that there exist an offspring distribution $\xi$ and a polynomial $Q(x)=b_{0}+\cdots+b_{r-2} x^{r-2}$ such that, for some positive constant $C$,

(1) $g_{\xi}(x)=C-x^{\nu} Q(x)$.

(2) $Q(x)>0$ for all $x \in[0,1]$.

This $\xi$, according to Theorem 3.5 and the fact that $b_{0}>0$, will indeed satisfy equation (3.5). We will find a sequence $\left\{\chi_{k}\right\}_{k=r}^{\infty}$ with a finite sum together with a polynomial $P(x)=a_{0}+\cdots+a_{r-2} x^{r-2}$, such that

(1) $g_{\chi}(x)=\sum_{k} \chi_{k} g_{k}(x)=1-x^{\nu} P(x)$.

(2) $\chi_{k} \geq 0$.

(3) $P(x)>0$ for all $x \in[0,1]$.

Taking $\xi=\frac{1}{\sum \chi_{k}} \chi_{k}$ will then conclude the proof.

Let

$$
\chi_{k}=\left\{\begin{array}{ll}
\frac{r-1}{k(k-1)} & r \leq k \leq \nu+r-1 \\
0 & k \geq \nu+r
\end{array},\right.
$$

so that $g_{\chi}(x)=\sum_{k=r}^{\nu+r-1} \frac{r-1}{k(k-1)} g_{k}(x)$. Using Claim 4.3, we may write

$$
g_{\chi}(x)=1-\sum_{k=\nu+r}^{\infty} \frac{r-1}{k(k-1)} g_{k}(x) .
$$

By Claim $4.1 g_{\chi}$ is a polynomial of degree $\nu+r-2$, therefore $\sum_{k=\nu+r}^{\infty} \frac{r-1}{k(k-1)} g_{k}(x)$ equals a polynomial of degree $\nu+r-2$. Using again Claim 4.1, we can define the polynomial

$$
P(x)=\sum_{k=\nu+r}^{\infty} \frac{r-1}{k(k-1)} \frac{g_{k}(x)}{x^{\nu}} .
$$

It is left to show that $P(x)>0$ for all $x \in[0,1]$. By equations (3.2) and (2.2), $P(x)$ is non-negative and could only vanish at $x=0$. But by Claim 4.1, $P(0)=\frac{r-1}{(\nu+r)(\nu+r-1)}\left(\frac{g_{\nu+r}(x)}{x^{\nu}}\right)_{x=0} \neq 0$. This concludes the first part.

Before moving on to the proof of the second part, we make a remark that would be useful in the following.

Remark 4.4. Note that, by Claim 4.2, we can define the projection Pr from the space of polynomials of degree at most $r+\nu-2$ to its subspace spanned by $x^{\nu}, \ldots, x^{\nu+r-2}$ with kernel spanned by $g_{r}(x), \ldots, g_{\nu+r-1}(x)$. Define also $M_{0}$ to be the map from the space of polynomials of degree at most $r-2$ to the space of polynomials of degree at most $r+\nu-2$ given by the multiplication by $x^{\nu}$. Then the first of the conditions on $P$ in the proof of the first part of Theorem 3.8 can be written as

$$
\operatorname{Pr} M_{0} P=\operatorname{Pr} 1 .
$$

Since $\operatorname{Pr} \circ M_{0}$ is bijective, this equation has a unique solution; and what we have shown in the proof is that this solution satisfies the necessary positivity conditions. 
Proof of Theorem 3.8 part 2: We will now prove the second part of the theorem. In analogy with the first one, we will find $\bar{\xi}, \bar{Q}(x)=\bar{b}_{0}+\cdots+\bar{b}_{r-2} x^{r-2}$ and $x_{1}>\cdots>x_{n}$ such that, for some positive constant $\bar{C}$,

(1) $g_{\bar{\xi}}(x)=\bar{C}-\left(x-x_{1}\right)^{2 \nu_{1}} \ldots\left(x-x_{n}\right)^{2 \nu_{n}} \bar{Q}(x)$.

(2) $\bar{Q}(x)>0$ for all $x \in[0,1]$.

Similarly to the previous part, we will look for $\left\{\bar{\chi}_{k}\right\}_{k=r}^{\nu+r-1}$ and $\bar{P}(x)=\bar{a}_{0}+\cdots+$ $\bar{a}_{r-2} x^{r-2}$ satisfying:

(1) $g_{\bar{\chi}}(x)=\sum_{k} \bar{\chi}_{k} g_{k}(x)=1-\left(x-x_{1}\right)^{2 \nu_{1}} \ldots\left(x-x_{n}\right)^{2 \nu_{n}} \bar{P}(x)$.

(2) $\bar{\chi}_{k}>0$.

(3) $\bar{P}(x)>0$ for all $x \in[0,1]$.

We stress that the inequality $\bar{\chi}_{k}>0$ is strict, which will allow us to use a perturbation argument. Indeed, choosing $\nu=2 \nu_{1}+\cdots+2 \nu_{n}, \chi_{k}$ (defined in equation (4.3)) is strictly positive for $r \leq k \leq \nu+r-1$. Since $P$ was required to be strictly positive, we may hope that also after adding a small perturbation $\left(x_{1}, \ldots, x_{n}\right)$ around 0 there still exists a positive solution $\bar{P}$. More precisely, let us denote by $M_{x_{1}, \ldots, x_{n}}$ the multiplication by $\left(x-x_{1}\right)^{2 \nu_{1}} \ldots\left(x-x_{n}\right)^{2 \nu_{n}}$, acting on the polynomials of degree at most $r-2$. In particular, for $x_{1}, \ldots, x_{n}=0$ this is $M_{0}$ defined in Remark 4.4. Then, we want to show that the solution of

$$
\operatorname{Pr} M_{x_{1}, \ldots, x_{n}} \bar{P}=\operatorname{Pr} 1
$$

satisfies the positivity conditions 2 and 3 . By continuity of the determinant, when $\left(x_{1}, \ldots, x_{n}\right)$ is in a small neighborhood of 0 the operator $\operatorname{Pr} M_{x_{1}, \ldots, x_{n}}$ is invertible. Moreover, in an even smaller neighborhood of 0 the polynomial $\left(\operatorname{Pr} M_{x_{1}, \ldots, x_{n}}\right)^{-1} \operatorname{Pr} 1$ will satisfy the positivity condition 3 - matrix inversion is continuous, and the set of polynomials satisfying this condition is open and contains $\left(\operatorname{Pr} M_{0}\right)^{-1} \operatorname{Pr} 1$ by the first part of the proof. Finally, since coordinate projections of $1-\left(x-x_{1}\right)^{2 \nu_{1}} \ldots(x-$ $\left.x_{n}\right)^{2 \nu_{n}}\left(\operatorname{Pr} M_{x_{1}, \ldots, x_{n}}\right)^{-1} \operatorname{Pr} 1$ with respect to the basis defined in Claim 4.2 are continuous in $\left(x_{1}, \ldots, x_{n}\right)$, and since for $\left(x_{1}, \ldots, x_{n}\right)=0$ condition 2 is satisfied, by taking $\left(x_{1}, \ldots, x_{n}\right)$ in a further smaller neighborhood of 0 we are guaranteed to find a polynomial $\bar{P}$ satisfying the required conditions.

\section{Remarks on two other phase transitions and open questions}

5.1. More discontinuities of $\phi_{t}$. Consider, for example, $r=2$ and $\xi_{k}=\frac{3}{5} \mathbb{1}_{k=2}+$ $\frac{2}{5} \mathbb{1}_{k=5}$. The function $g_{\xi}(x)$ is maximal at $g_{\xi}(0)=\frac{6}{5}$, then it has a local minimum, followed by a local maximum (see Figure 5.2). In this case, recalling Fact $3.2, \phi_{t}^{\xi}$ will have a discontinuity at this local maximum, that is, a second phase transition occurs. We may then expect that one can find $\xi$ giving rise to as many (decreasing) local maxima of $g_{\xi}$ as we wish:

Conjecture 5.1. Let $\nu_{1}^{(1)}, \ldots, \nu_{n_{1}}^{(1)}, \nu_{1}^{(2)}, \ldots, \nu_{n_{2}}^{(2)}, \ldots, \nu_{n_{m}}^{(m)}$. Then there exist $\xi$, $\left\{q_{i}\right\}_{i=1}^{m},\left\{x_{j}^{(i)}\right\}_{1<i<m, 1<j<n_{i}}$ such that $q_{i}$ is a critical point which is $\left(\nu_{1}^{(i)}, \ldots, \nu_{n_{i}}^{(i)}\right)$ metastable at $x_{1}^{(\bar{i})}, \ldots, x_{n_{i}}^{(i)}$.

5.2. Percolation of infection. Another possible phase transition, studied in Fontes and Schonmann (2008) for the case of regular trees, is when infinite infected clusters start to appear, but the prevalence is still smaller than 1. Following the proof of 


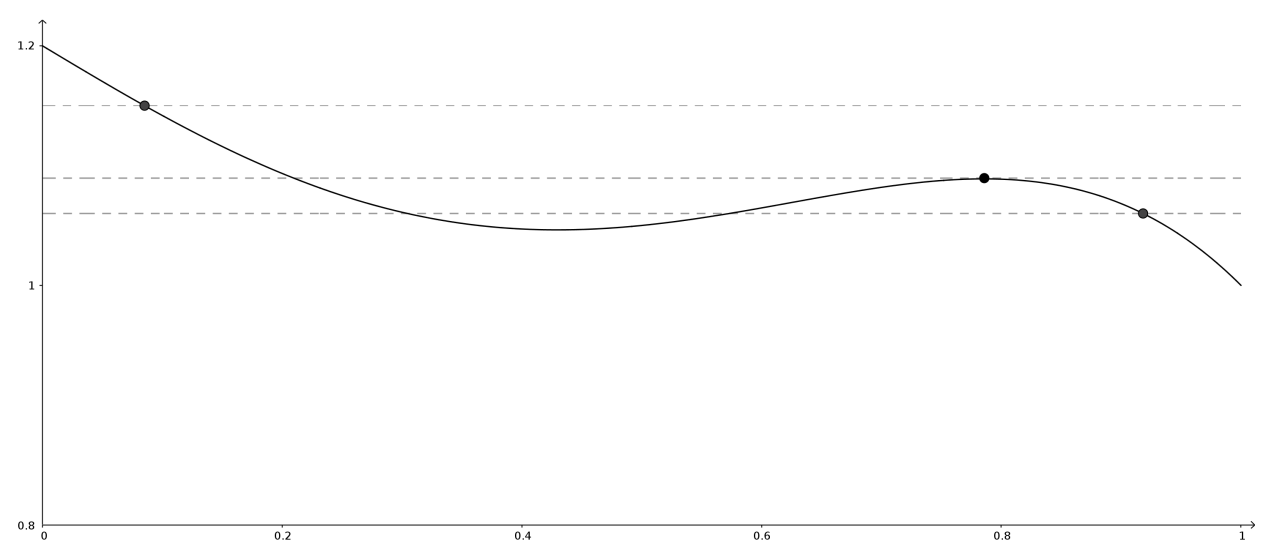

FiguRE 5.2. $g_{\xi}$ for $r=2$ and $\xi_{k}=\frac{3}{5} \mathbb{1}_{k=2}+\frac{2}{5} \mathbb{1}_{k=5}$. We show three lines $\frac{1}{q}$ for three parameters $q$, intersecting $g_{\xi}$ at $\phi_{\infty}^{\xi}$. One sees here the discontinuity when $\frac{1}{q}$ equals the value of $g_{\xi}$ at the local maximum.

Proposition 3.9 in Fontes and Schonmann (2008), one sees that it applies also for the bootstrap percolation on GW trees, showing that the critical probability $q_{c}^{(\infty)}$ above which infinite clusters no longer appear is strictly bigger than $q_{c}$ defined in equation (3.1), unless $\xi_{k}=\mathbb{1}_{r}$.

5.3. More questions. The problem of bootstrap percolation in disordered systems raises many questions. Related to the work presented here, one may be interested in the metastable regime for other systems, such as $G_{n, p}$ or the random regular graph. Another natural problem is the analysis of the bootstrap percolation on the random graph with a given degree sequence, that has a GW local structure, with analogy to the regular tree structure of the random regular graph.

\section{Acknowledgments}

I would like to thank Cristina Toninelli for the introduction of the subject and for useful discussion, and to Lucas Benigni for his help throughout the writing of this paper.

\section{Appendix A. Appendix}

This paper concerns the analysis of a phase transition originating in the appearance of a new fixed point for a certain recurrence relation, i.e., a bifurcation. In this appendix, we will try to understand in a more general context the time scaling in systems of that type. Let us then consider a sequence of reals $\left\{x_{n}\right\}_{n=0}^{\infty}$, defined by the value $x_{0}$ and a recursion formula for $n>0$ :

$$
x_{n}=f\left(x_{n-1}\right) .
$$

We will also fix now some positive $\delta \in(0,1)$, that will be used throughout this appendix as the window around the new fixed point in which we are interested. 
First, we will study the time scaling at the bifurcation point, when the new fixed point is first created. In this case, we may expect $f$ to be tangent to the identity function at the fixed point, so we will start our discussion with the following assumptions on $f$ and $\delta$ :

Assumption A.1. $f$ has a fixed point $y_{0}$, such that for $y \in\left(y_{0}, y_{0}+\delta\right)$ :

$$
y-\underline{c}\left(y-y_{0}\right)^{\alpha} \leq f(y) \leq y-\bar{c}\left(y-y_{0}\right)^{\alpha} \text {, }
$$

for some $\alpha>1,0<\bar{c} \leq \underline{c}<\delta^{-(\alpha-1)}$.

Assumption A.2. $x_{0} \in\left(y_{0}, y_{0}+\delta\right)$.

We first mention the following fact:

Fact A.3. Under the assumptions $A .1$ and $A .2$ the sequence $\left(x_{n}\right)$ is decreasing and bounded from below by $y_{0}$.

Proof: By Assumption A.1, $x_{n+1}<x_{n}$ whenever $x_{n} \in\left(y_{0}, y_{0}+\delta\right)$. Moreover,

$$
\begin{aligned}
x_{n+1}-y_{0} & \geq x_{n}-y_{0}-\underline{c}\left(x_{n}-y_{0}\right)^{\alpha} \\
& =\left(x_{n}-y_{0}\right)\left(1-\underline{c}\left(x_{n}-y_{0}\right)^{\alpha-1}\right) \\
& \geq\left(x_{n}-y_{0}\right)\left(1-\underline{c} \delta^{\alpha-1}\right) \\
& >0 .
\end{aligned}
$$

Therefore, since $x_{0} \in\left(y_{0}, y_{0}+\delta\right)$ by assumption A.2, the entire sequence is in the interval $\left(y_{0}, y_{0}+\delta\right)$, and it is decreasing.

The following theorem will describe the asymptotic of the sequence:

Theorem A.4. Let $\left\{x_{n}\right\}_{n=0}^{\infty}$ be the sequence defined in equation (A.1), satisfying Assumptions A.1 and A.2 for some $\delta \in(0,1)$. Then

$$
y_{0}+\underline{a}\left(n+n_{0}\right)^{-\frac{1}{\alpha-1}} \leq x_{n} \leq y_{0}+\bar{a} n^{-\frac{1}{\alpha-1}},
$$

where $\underline{a}=\left[(\alpha-1)(1-\delta)^{-\alpha} \underline{c}\right]^{-\frac{1}{\alpha-1}}, \bar{a}=[(\alpha-1) \bar{c}]^{-\frac{1}{\alpha-1}}$, and $n_{0}=\frac{\left(x_{0}-y_{0}\right)^{1-\alpha}}{(\alpha-1)(1-\delta)^{-\alpha} \underline{c}}$ are all positive constants.

Proof: Let us first define a sequence $t_{n}=\left(x_{n}-y_{0}\right)^{1-\alpha}$, and note that $t_{n}$ is positive for all $n$. Then using Fact A.3 and Assumption A.1, fixing $\underline{c}^{\prime}=(\alpha-1)(1-\delta)^{-\alpha} \underline{c}$ and $\bar{c}^{\prime}=(\alpha-1) \bar{c}$, we can estimate:

$$
\begin{aligned}
t_{n} & =\left(f\left(x_{n-1}\right)-y_{0}\right)^{1-\alpha} & t_{n} & =\left(f\left(x_{n-1}\right)-y_{0}\right)^{1-\alpha} \\
& \leq\left(x_{n-1}-\underline{c}\left(x_{n-1}-y_{0}\right)^{\alpha}-y_{0}\right)^{1-\alpha} & & \geq\left(x_{n-1}-\bar{c}\left(x_{n-1}-y_{0}\right)^{\alpha}-y_{0}\right)^{1-\alpha} \\
& =\left(t_{n-1}^{\frac{1}{1-\alpha}}-\underline{c} t_{n-1}^{\frac{\alpha}{1-\alpha}}\right)^{1-\alpha} & & =\left(t_{n-1}^{\frac{1}{1-\alpha}}-\bar{c} t_{n-1}^{\frac{\alpha}{1-\alpha}}\right) \\
& =t_{n-1}\left(1-\underline{c} t_{n-1}^{-1}\right)^{1-\alpha} & & =t_{n-1}\left(1-\bar{c} t_{n-1}^{-1}\right)^{1-\alpha} \\
& \leq t_{n-1}\left(1+\underline{c}^{\prime} t_{n-1}^{-1}\right) & & \geq t_{n-1}\left(1+\bar{c}^{\prime} t_{n-1}^{-1}\right) \\
& =t_{n-1}+\underline{c}^{\prime} ; & & =t_{n-1}+\bar{c}^{\prime} .
\end{aligned}
$$

We have used here the fact that, for any $0<z<\delta<1$, we can approximate $(1-z)^{1-\alpha}$ using its derivatives at 0 and at $\delta$ :

$$
-(1-\alpha) \leq \frac{(1-z)^{1-\alpha}-1}{z} \leq-(1-\alpha)(1-\delta)^{-\alpha} .
$$


We then also use $\underline{c} t_{n-1}^{-1}=\left(x_{n}-y_{0}\right)^{\alpha-1}<\delta^{\alpha-1}<\delta$.

Finally,

$$
\begin{aligned}
x_{n} & =y_{0}+t_{n}^{-\frac{1}{\alpha-1}} & & x_{n} \geq y_{0}+\left(\left(x_{0}-y_{0}\right)^{1-\alpha}+\underline{c}^{\prime} n\right)^{-\frac{1}{\alpha-1}} \\
& \leq y_{0}+\left(\left(x_{0}-y_{0}\right)^{1-\alpha}+\bar{c}^{\prime} n\right)^{-\frac{1}{\alpha-1}} & & =y_{0}+\left(\underline{c}^{\prime}\left(n+\frac{\left(x_{0}-y_{0}\right)^{1-\alpha}}{\underline{c}^{\prime}}\right)\right)^{-\frac{1}{\alpha-1}} \\
& \leq y_{0}+\bar{a} n^{-\frac{1}{\alpha-1}} ; & & =y_{0}+\underline{a}\left(n+n_{0}\right)^{-\frac{\frac{1}{\alpha-1}}{\alpha}} .
\end{aligned}
$$

Next, we will be interested in the behavior near the bifurcation point, just before the new fixed point appears. For this purpose we will consider a family $\left\{x_{n}^{\varepsilon}\right\}_{n=0}^{\infty}$ of sequences, each defined by the value $x_{0}^{\varepsilon}$ and a recursion formula for $n>0$ :

$$
x_{n}^{\varepsilon}=f_{\varepsilon}\left(x_{n-1}^{\varepsilon}\right) \text {, }
$$

and assume:

Assumption A.5. There is a point $y_{0}$ such that for $\left|y-y_{0}\right|<\delta$ and $\varepsilon<\varepsilon_{0}$

$$
y-\underline{c}\left(y-y_{0}\right)^{2 \alpha}-\varepsilon \leq f_{\varepsilon}(y) \leq y-\bar{c}\left(y-y_{0}\right)^{2 \alpha}-\theta \varepsilon,
$$

for an integer $\alpha>1$, positive constants $\bar{c}$ and $\underline{c}$, and some fixed $\theta \in(0,1]$.

Assumption A.6. $0<x_{0}-y_{0}<\delta$.

In order to study the asymptotic behavior of $x_{n}^{\varepsilon}$ for small values of $\varepsilon$, we will need the following definition:

Definition A.7. The exit time $N_{\delta}(\varepsilon)$ is the minimal $n$ such that $x_{n}^{\varepsilon}<y_{0}-\delta$.

Replacing Fact A.3 will be the following:

Fact A.8. For all $\varepsilon<\varepsilon_{0}, N_{\delta}(\varepsilon)$ is finite, and for $n<N_{\delta}(\varepsilon)$ the sequence $x_{n}^{\varepsilon}$ is decreasing.

Proof: By Assumption A.5, for $n<N_{\delta}(\varepsilon)$, if $x_{n}^{\varepsilon}<y_{0}+\delta$ then $x_{n+1}^{\varepsilon}<x_{n}^{\varepsilon}<$ $y_{0}+\delta$. Hence, the sequence remains in the interval $\left(y_{0}-\delta, y_{0}+\delta\right)$ an long as $n<N_{\delta}(\varepsilon)$. Since in this interval the sequence is decreasing, the result follows by Assumption A.6.

For our analysis, we will compare this sequence to the solution of the following differential equations, that will approximate $x_{n}^{\varepsilon}-y_{0}$ :

$$
\begin{aligned}
\frac{\mathrm{d} \zeta}{\mathrm{d} s} & =-\underline{c} \underline{\zeta}^{2 \alpha}-\varepsilon, \quad \underline{\mathrm{d} \bar{\zeta}}=-\bar{c} \bar{\zeta}^{2 \alpha}-\theta \varepsilon, \\
\underline{\zeta}(0) & =z_{0}^{\varepsilon}=x_{0}^{\varepsilon}-y_{0} ; \quad \bar{\zeta}(0)=z_{0}^{\varepsilon}=x_{0}^{\varepsilon}-y_{0} .
\end{aligned}
$$

The solution $\bar{\zeta}$ is strictly decreasing, and in particular one can define its inverse $\bar{t}:\left[-\infty, z_{0}^{\varepsilon}\right] \rightarrow[0, \infty]$, and $\bar{\tau}_{n}=\bar{t}\left(x_{n}^{\varepsilon}-y_{0}\right) . \underline{t}$ and $\underline{\tau}_{n}$ will be defined in the same manner. Note that these all depend on $\varepsilon$, even though this dependence is omitted from the notation. The next lemma will show that the continuous crossing times $\bar{\tau}_{n}$ and $\underline{\tau}_{n}$ are close to the discrete one, namely $n$.

Lemma A.9. For all $n \leq N_{\delta}(\varepsilon)$,

$$
\left(1-\kappa_{\bar{c}, \delta, \theta \varepsilon}\right) n \leq \bar{\tau}_{n} \leq \underline{\tau}_{n} \leq\left(1+\kappa_{\underline{c}, \delta, \varepsilon}\right) n,
$$

where for all $c>0, \kappa_{c, \delta, \varepsilon}=\max \left(C_{4} \varepsilon^{2 \alpha-1}, 2 \alpha \delta^{2 \alpha-1}\right) . C_{4}$ is a positive constant depending on $\delta, c$ and $\varepsilon_{0}$ given explicitly in the proof, and bounded when $\delta$ and $\varepsilon_{0}$ are not too big. For example, if $\varepsilon_{0}<1$ and $c \delta^{2 \alpha-1}<\frac{1}{2}, C_{4}<\left(3+4^{\alpha} c\right)^{4 \alpha}$. 
Proof: Let $z_{n}=x_{n}-y_{0}$. Then

$$
\begin{aligned}
\underline{\tau}_{n} & =\underline{t}\left(f_{\varepsilon}\left(x_{n-1}\right)-y_{0}\right) \\
& \leq \underline{t}\left(z_{n-1}-\underline{c} z_{n-1}^{2 \alpha}-\varepsilon\right) \\
& =\int_{z_{0}}^{z_{n-1}-\underline{c} z_{n-1}^{2 \alpha}-\varepsilon} \frac{\mathrm{d} z}{-\underline{c} z^{2 \alpha}-\varepsilon} \\
& =\underline{t}\left(z_{n-1}\right)-\int_{z_{n-1}}^{z_{n-1}-\underline{c} z_{n-1}^{2 \alpha}-\varepsilon} \frac{\mathrm{d} z}{z_{n-1}-\underline{c} z_{n-1}^{2 \alpha}+\varepsilon}-\int_{z_{n-1}^{2 \alpha}-\varepsilon}^{z_{n-1}-c z_{n-1}^{2 \alpha}-\varepsilon}\left(\frac{\mathrm{d} z}{\underline{c} z^{2 \alpha}+\varepsilon}-\frac{\mathrm{d} z}{\underline{c} z_{n-1}^{2 \alpha}+\varepsilon}\right) \\
& =\underline{\tau}_{n-1}+1-\int_{z_{n-1}}\left(\frac{\mathrm{d} z}{\underline{c} z^{2 \alpha}+\varepsilon}-\frac{\mathrm{d} z}{\underline{c} z_{n-1}^{2 \alpha}+\varepsilon}\right) .
\end{aligned}
$$

In order to study the error term, we will use the following estimation:

Claim A.10. Fix $w_{0} \in(-\delta, \delta)$, and $c>0$. Let

$$
I=\int_{w_{0}-c w_{0}^{2 \alpha}-\varepsilon}^{w_{0}}\left(\frac{1}{c w^{2 \alpha}+\varepsilon}-\frac{1}{c w_{0}^{2 \alpha}+\varepsilon}\right) d w .
$$

Then

$$
|I| \leq \kappa_{c, \delta, \varepsilon_{0}} .
$$

Proof: We will first consider the case in which the integration interval passes through 0 , that is $0<w_{0}<c w_{0}^{2 \alpha}+\varepsilon$. In this case,

$$
\begin{aligned}
w_{0} & \leq w_{0}\left(1-c w_{0}^{2 \alpha-1}\right)\left(1-c \delta^{2 \alpha-1}\right)^{-1} \leq C_{1} \varepsilon, \\
c w_{0}^{2 \alpha}+\varepsilon & \leq\left[1+C_{2} \varepsilon^{2 \alpha-1}\right] \varepsilon,
\end{aligned}
$$

for $C_{1}=\left(1-c \delta^{2 \alpha-1}\right)^{-1}$ and $C_{2}=c\left(1-c \delta^{2 \alpha-1}\right)^{-2 \alpha}$.

We may then, for all for all $w \in\left[w_{0}-c w_{0}^{2 \alpha}-\varepsilon, w_{0}\right]$, bound the nominator of the integrand by

$$
\left|c w_{0}^{2 \alpha}+\varepsilon-c w^{2 \alpha}-\varepsilon\right| \leq c w_{0}^{2 \alpha}+c w^{2 \alpha} \leq C_{3} \varepsilon^{2 \alpha},
$$

where $C_{3}=\left(1+C_{2} \varepsilon_{0}^{2 \alpha-1}\right)^{2 \alpha}+C_{1}^{2 \alpha}$.

For the denominator,

$$
\left(c w^{2 \alpha}+\varepsilon\right)\left(c w_{0}^{2 \alpha}+\varepsilon\right) \geq \varepsilon^{2} .
$$

Putting everything together

$$
\begin{aligned}
|I| & \leq \int_{w_{0}-c w_{0}^{2 \alpha}-\varepsilon}^{w_{0}}\left|\frac{c w_{0}^{2 \alpha}+\varepsilon-c w^{2 \alpha}-\varepsilon}{\left(c w^{2 \alpha}+\varepsilon\right)\left(c w_{0}^{2 \alpha}+\varepsilon\right)}\right| \\
& \leq\left(c w_{0}^{2 \alpha}+\varepsilon\right) C_{3} \varepsilon^{2 \alpha-2} \\
& \leq C_{4} \varepsilon^{2 \alpha-1}
\end{aligned}
$$

for $C_{4}=\left[1+C_{2} \varepsilon_{0}^{2 \alpha-1}\right] C_{3}$. 
Next, we consider the case where the integral is over a positive interval, i.e., $w_{0} \geq c w_{0}^{2 \alpha}+\varepsilon$. We can bound the integrand using convexity - for all $w \in$ $\left(w_{0}-c w_{0}^{2 \alpha}-\varepsilon, w_{0}\right)$

$$
\frac{\frac{1}{c w^{2 \alpha}+\varepsilon}-\frac{1}{c w_{0}^{2 \alpha}+\varepsilon}}{w-w_{0}} \geq-\frac{2 \alpha c w^{2 \alpha-1}}{\left(c w^{2 \alpha}+\varepsilon\right)^{2}}
$$

This implies that

$$
\begin{aligned}
|I| & \leq\left(c w_{0}^{2 \alpha}+\varepsilon\right) \frac{2 \alpha c w^{2 \alpha-1}}{\left(c w^{2 \alpha}+\varepsilon\right)^{2}}\left(w_{0}-w\right) \\
& \leq 2 \alpha c w^{2 \alpha-1} \leq 2 \alpha c \delta^{2 \alpha-1} .
\end{aligned}
$$

We are left with the case $w_{0} \leq-c w_{0}^{2 \alpha}-\varepsilon$, which could be analyzed using the exact same argument as the previous one to obtain the result.

Using the claim we can continue with our estimation, obtaining

$$
\underline{\tau}_{n} \leq \underline{\tau}_{n-1}+1+\kappa_{\underline{c}, \delta, \varepsilon_{0}},
$$

and proving the upper bound. The lower bound could be found using the exact same calculation replacing $\underline{c}$ by $\bar{c}$ and $\varepsilon$ by $\theta \varepsilon$. The result follows since $\bar{c} \leq \underline{c}$, and thus $\bar{\tau}_{n} \leq \underline{\tau}_{n}$ by monotonicity of the integral.

We are now ready to formulate the final result:

Theorem A.11. Fix a family of sequences (indexed by $\varepsilon$ ) defined in equation (A.2) satisfying Assumptions A.5 and A.6, and consider their exit times $N_{\delta}(\varepsilon)$ (see Definition A.7). Let $\bar{I}=\int_{-\infty}^{\infty} \frac{d u}{\bar{c} u^{2 \alpha}+1}, \underline{I}=\int_{-\infty}^{\infty} \frac{d u}{\underline{c} u^{2 \alpha}+1}$, and $\kappa_{\delta, 0}=\max \left(\kappa_{\bar{c}, \delta, 0}, \kappa_{\underline{c}, \delta, 0}\right)$, where $\kappa_{\bar{c}, \delta, 0}$ and $\kappa_{\underline{c}, \delta, 0}$ are the positive constants given in Lemma A.9. Assume further that $\kappa_{\delta, 0}<1$. Then

$$
0<\frac{\frac{1}{2} \underline{I}}{1+\kappa_{\delta, 0}} \leq \liminf _{\varepsilon \rightarrow 0} \frac{N_{\delta}(\varepsilon)}{\varepsilon^{-1+\frac{1}{2 \alpha}}} \leq \limsup _{\varepsilon \rightarrow 0} \frac{N_{\delta}(\varepsilon)}{\varepsilon^{-1+\frac{1}{2 \alpha}}} \leq \frac{\bar{I}}{\theta\left(1-\kappa_{\delta, 0}\right)}<\infty .
$$

The factor $\frac{1}{2}$ in front of $\underline{I}$ could be removed when $\varepsilon^{-\frac{1}{2 \alpha}}\left(x_{0}^{\varepsilon}-y_{0}\right)^{\varepsilon} \rightarrow \infty$ as $\varepsilon \rightarrow 0$ (e.g., when $x_{0}^{\varepsilon}-y_{0}$ is bounded away from 0 uniformly in $\varepsilon$ ).

Proof: This theorem is a direct consequence of the fact that $\zeta$ shows an $\varepsilon^{-1+\frac{1}{2 \alpha}}$ time scaling behavior. First, note that

$$
\bar{\tau}_{N_{\delta}(\varepsilon)-1} \leq \bar{t}(-\delta) \leq \underline{t}(-\delta) \leq \underline{\tau}_{N_{\delta}(\varepsilon)} .
$$

We will then be interested in finding $\bar{t}(-\delta), \underline{t}(-\delta)$ :

$\underline{t}(-\delta)=\int_{z_{0}^{\varepsilon}}^{-\delta} \frac{\mathrm{d} z}{-\underline{c} z^{2 \alpha}-\varepsilon}=\varepsilon^{-1+\frac{1}{2 \alpha}} \int_{z_{0}^{\varepsilon}}^{-\delta} \frac{\varepsilon^{-\frac{1}{2 \alpha}} \mathrm{d} z}{-\underline{c}\left(z \varepsilon^{-\frac{1}{2 \alpha}}\right)^{2 \alpha}-1}=\varepsilon^{-1+\frac{1}{2 \alpha}} \int_{-\varepsilon^{-\frac{1}{2 \alpha}}}^{z_{0}^{\varepsilon}} \frac{\mathrm{d} u}{\underline{c} u^{2 \alpha}+1}$,

and for $\bar{t}$ one should replace $\underline{c}$ by $\bar{c}$ and $\varepsilon$ by $\theta \varepsilon$.

All that is left is to use Lemma A.9, finding

$$
\frac{\underline{t}(-\delta)}{\left(1+\kappa_{\delta, \varepsilon}\right)} \leq N_{\delta}(\varepsilon) \leq 1+\frac{\bar{t}(-\delta)}{\left(1-\kappa_{\delta, \varepsilon}\right)}
$$


which, since the integrals defining $\bar{I}$ and $\underline{I}$ converge, concludes the proof.

Remark A.12. When $f_{\varepsilon}$ satisfies not only Assumption A.5, but also

$$
f_{\varepsilon}(y)=y-c\left(y-y_{0}\right)^{2 \alpha}-\varepsilon+o\left(\left(y-y_{0}\right)^{2 \alpha}\right)+o(\varepsilon),
$$

we can consider $\delta_{\varepsilon}$ that goes to 0 with $\varepsilon$, e.g. $\frac{1}{|\log \varepsilon|}$, so that $\kappa_{\delta, 0}$ will converge to 0 as well. In this case, we may choose $\bar{c}_{\delta}$ and $\underline{c}_{\delta}$ that converge to $c$, and thus Theorem A.11 will give the limit of $\frac{N_{\delta}(\varepsilon)}{\varepsilon^{-1+\frac{1}{2 \alpha}}}$, rather than just bounds on its limsup and liminf. Such a direct application of the theorem, however, forces us to choose an initial condition $x_{0}^{\varepsilon}$ that converges to $y_{0}$ as $\varepsilon$ goes to 0 . To overcome this issue, we can use the estimation above with a fixed $\delta$ until $x_{n}$ reaches $\delta_{\varepsilon}$, which happens at $n$ of order $\int_{z_{0}}^{\delta_{\varepsilon}} \frac{\mathrm{d} z}{-c z^{2 \alpha}-\varepsilon} \ll \varepsilon^{-1+\frac{1}{2 \alpha}}$. Then restart the dynamics using the estimation with $\delta_{\varepsilon}$ until reaching $-\delta_{\varepsilon}$, which takes an order $\varepsilon^{-1+\frac{1}{2 \alpha}}$ of steps, and then using again the estimation for our fixed $\delta$ show that the number of steps required to reach $-\delta$ is much smaller than $\varepsilon^{-1+\frac{1}{2 \alpha}}$. This would yield

$$
\lim _{\varepsilon \rightarrow 0} \frac{N_{\delta}(\varepsilon)}{\varepsilon^{-1+\frac{1}{2 \alpha}}}=\int_{-\infty}^{\infty} \frac{\mathrm{d} u}{c u^{2 \alpha}+1} .
$$

Lemma A.13. Fix a family of sequences (indexed by $\varepsilon$ ) defined in equation (A.2). Assume that there exist $n$ points $y_{1}, \ldots, y_{n}, n$ exponents $\alpha_{1}, \ldots, \alpha_{n}$ and $\delta>0$ such that, for $\varepsilon$ small enough,

(1) $x_{0}>y_{1}$

(2) For all $i \leq n$, as $y \rightarrow y_{i}$ and $\varepsilon \rightarrow 0$

$$
f_{\varepsilon}(y)=y-c\left(y-y_{i}\right)^{2 \alpha_{i}}-\varepsilon+o\left(\left(y-y_{i}\right)^{2 \alpha_{i}}+\varepsilon\right) .
$$

(3) There exists $a>0$ that may depend on $\delta$ but not on $\varepsilon$, such that if $\left|y-y_{i}\right| \geq \delta$ for all $i$ then $f_{\varepsilon}(y)<y-a$.

Let $\tau_{i}^{-}=\min \left(n: x_{n}<y_{i}+\delta\right)$ and $\tau_{i}^{+}=\min \left(n: x_{n}<y_{i}-\delta\right)$. Then

(1) $\tau_{1}<a\left(x_{0}-y_{1}\right)$,

(2) $\frac{\log \left(\tau_{i}^{+}-\tau_{i}^{-}\right)}{\log (\varepsilon)} \rightarrow-1+\frac{1}{2 \alpha_{i}}$ as $\varepsilon \rightarrow 0$,

(3) $\tau_{i+1}^{-}-\tau_{i}^{+}<a\left(y_{i}-y_{i+1}\right)$.

Proof: Parts 1 and 3 are an immediate consequence of the assumptions 1 and 3 . Part 2 is a consquence of Theorem A.11 - for any fixed $i$, when starting from $x_{\tau_{i}^{-}}$assumptions A.6 and A.5 are satisfied. The result follows by noting that $\tau^{+^{2}}-\tau^{-}=N_{\delta}(\varepsilon)$.

\section{References}

M. Aizenman and J. L. Lebowitz. Metastability effects in bootstrap percolation. J. Phys. A 21 (19), 3801-3813 (1988). MR968311.

J. Balogh, B. Bollobás, H. Duminil-Copin and R. Morris. The sharp threshold for bootstrap percolation in all dimensions. Trans. Amer. Math. Soc. 364 (5), 2667-2701 (2012). MR2888224.

J. Balogh, Y. Peres and G. Pete. Bootstrap percolation on infinite trees and nonamenable groups. Combin. Probab. Comput. 15 (5), 715-730 (2006). MR2248323. 
J. Balogh and Boris G. Pittel. Bootstrap percolation on the random regular graph. Random Structures Algorithms 30 (1-2), 257-286 (2007). MR2283230.

M. Biskup and R. H. Schonmann. Metastable behavior for bootstrap percolation on regular trees. J. Stat. Phys. 136 (4), 667-676 (2009). MR2540158.

B. Bollobás, K. Gunderson, C. Holmgren, S. Janson and M. Przykucki. Bootstrap percolation on Galton-Watson trees. Electron. J. Probab. 19, no. 13, 27 (2014). MR3164766.

J. Chalupa, P. L Leath and G. R Reich. Bootstrap percolation on a Bethe lattice. Journal of Physics C: Solid State Physics 12 (1), L31 (1979).

L. R. G. Fontes and R. H. Schonmann. Bootstrap percolation on homogeneous trees has 2 phase transitions. J. Stat. Phys. 132 (5), 839-861 (2008). MR2430783.

J. Gravner and A. E. Holroyd. Polluted bootstrap percolation with threshold two in all dimensions. Probab. Theory Related Fields 175 (1-2), 467-486 (2019). MR4009714.

J. Gravner and E. McDonald. Bootstrap percolation in a polluted environment. $J$. Statist. Phys. 87 (3-4), 915-927 (1997). MR1459046.

A. E. Holroyd. Sharp metastability threshold for two-dimensional bootstrap percolation. Probab. Theory Related Fields 125 (2), 195-224 (2003). MR1961342.

S. Janson. On percolation in random graphs with given vertex degrees. Electron. J. Probab. 14, no. 5, 87-118 (2009). MR2471661.

S. Janson, T. Łuczak, T. Turova and T. Vallier. Bootstrap percolation on the random graph $G_{n, p}$. Ann. Appl. Probab. 22 (5), 1989-2047 (2012). MR3025687. 